\title{
AiMT
}

Advances in Military Technology

Vol. 15, No. 2, 2020, pp. 231-246

ISSN 1802-2308, eISSN 2533-4123

DOI 10.3849/aimt.01376

\section{Development of a Method for Boundary Determination of the Noise-resistant Area of the UHF/VHF Band}

\author{
O. Iohov ${ }^{1}$, V. Maliuk ${ }^{1}$, S. Horielyshev ${ }^{1 *}$, \\ K. Tkachenko ${ }^{1}$ and S. Herasimov ${ }^{2}$ \\ ${ }^{1}$ National Academy of National Guard of Ukraine, Kharkiv, Ukraine \\ ${ }^{2}$ Ivan Kozhedub Kharkiv National Air Force University, Kharkiv, Ukraine
}

The manuscript was received on 15 January 2020 and was accepted after revision for publication as research paper on 24 July 2020.

\begin{abstract}
:
A method is proposed for determining the boundaries of the maximum largest zone of stable radio reception in the UHF/VHF range using beam antenna system under the action of several sources of radio interference located at different altitudes. The increase in the size of the stable radio reception area is ensured by the optimal orientation of the antenna arrangement at each point in azimuth and angular altitude using the radio communication channel model. To determine the coordinates of the map points belonging to the boundary, a modification of the wave algorithm is proposed. The conducted experimental studies for the Mototrbo ${ }^{T M}$ DP4800 and Kenwood TK-8302 radio equipment allow concluding that the theoretical positions and computer simulation analysis results obtained are reliable.
\end{abstract}

\section{Keywords:}

defensive electronic countermeasures, interference immunity, isoline, wavelet algorithm

\section{Introduction}

An important area of research in current conflicts is the confrontation of electronic systems. In the context of the rapid development of electronic warfare (EW), a set of urgent problems arose in the field of providing necessary indicators of the stability of communication systems, in particular noise-resistant and electromagnetic compatibility (EMC) of electronic means. One of the most effective means of EW is the setting of intentional interference, allowing to simultaneously perform the task of blocking access to radiotelephony means and to violate message integrity [1-4].

\footnotetext{
* Corresponding author: Scientific and Research Centre of Service and Military Activities of the National Guard of Ukraine, Kharkiv, National Academy of National Guard of Ukraine, Zakhysnykiv Ukrainy sq., 3, Ukraine.Phone:+380 6795991 53, E-mail: port_6633@ukr.net
} 
In mobile communication system, the tasks of protecting the UHF/VHF band of mobile radio communication equipment, office and fieldbus networks from industrial radio interference, overhead transmission lines and high-voltage equipment interference are similar [5-7].

Thus, the relevant objective is to determine the boundaries of the area of noiseresistant radio communication with a maximum size.

Potential existence of several interferers, as well as a possibility of using screens and directional antenna systems for protection radiotelephony means from the effects of such sources should be used in calculations.

The existing powerful commercial systems, such as, for example, HTZ WARFARE by ATDI [8] and EMC ANALYSIS by ALTAIR [9], among others, can solve the problem of predicting the characteristics of radiation and calculating EMC. At the same time, such software products have a high cost of their acquisition, closed software, and redundancy of functions relative to the particular task under consideration. Therefore, it is necessary to obtain a simple and effective method for calculating the boundaries of the maximum area of stable radio reception in the UHF/VHF band for mobile radio communication equipment under conditions of radio interference system.

Knowing the threshold signal-to-noise ratio $S N R_{\min }$, as an objective assessment of the level of protection of the radio receiver against interference, we can take the coefficient of suppression of the useful signal power by interference with a known limit (threshold) signal-to-noise ratio [10]:

$$
K_{\text {sup }}=\frac{1}{S N R}=\frac{P_{\mathrm{N}}}{P_{\mathrm{S}}} \leq K_{\mathrm{th}}
$$

where $K_{\mathrm{th}}=\frac{1}{S N R_{\min }}-$ the threshold signal, which depends on countermeasure and signal kind, the conditions of their interaction and the method of processing the sum of the signal and countermeasure;

$P_{\mathrm{N}}-$ the power of interference signal,

$P_{\mathrm{S}}-$ the power of desired signal.

If $\Omega$ is the area on the map in which the objects of radiotelephony can be located, then the area $\Omega_{\mathrm{s}} \in \Omega$ can be considered the area of noise-resistant radiotelephony, at any point of which the following relation holds Eq. (1):

$$
\Omega_{\mathrm{s}}=\left\{\forall(x, y) \in \Omega \mid K_{\text {sup }} \leq K_{\text {th }}\right\}
$$

Accordingly, the problem of increasing the size of area Eq. (2) can be written as the problem of maximizing the coverage coefficient of an area on the map

$$
K_{\mathrm{cov}}=\frac{\Omega_{\mathrm{s}}}{\Omega} \rightarrow \max
$$

Consider the known approaches to solving tasks Eq. (2) and Eq. (3) of calculating the boundaries and increasing the size of area $\Omega_{\text {s. }}$.

In the study [1], an analytical method for determining the boundaries of area $\Omega_{\mathrm{s}}$ for the case of a single source of radio interference is given in Fig. 1.

The reduction ratio Eq. (1) of the transmitter desired signal at the input of the receiver is obtained in the following form; 


$$
K_{\text {sup }}=\frac{P_{\mathrm{N}}}{P_{\mathrm{S}}}=\frac{P_{3} G_{32} G_{23} \eta_{\mathrm{N}} R_{12}^{2}}{P_{1} G_{12} G_{21} R_{23}^{2}}
$$

where $P_{1}$ - is the transmitted power of desired signal,

$P_{3}-$ is the power of interfering transmitter,

$G_{12}$ and $G_{21}$ - is the effective antenna gain of signal transmitter in the direction to the receiver and the receiving antenna in the direction to the transmitter,

$G_{32}$ and $G_{23}$ - is the effective antenna gain of interfering transmitter in the direction to the receiver and the receiving antenna in the direction to the interfering transmitter,

$R_{12}$ - the length of the propagation desired radio signal path, in direct visibility conditions, is equal to the distance from the transmitter to the receiver,

$R_{23}$ - the length of the propagation radio interference,

$\eta_{\mathrm{N}} \leq 1-$ the coefficient that takes into account the difference in signal polarization and interference.

It is shown that the form of the $\Omega_{\mathrm{s}}$ area depends on the power ratio of the transmitters $P_{1}$ and $P_{3}$. The authors did not consider the possibility of increasing the number of interfering sources. Also, the possibility of increasing the size of $\Omega_{\mathrm{s}}$ area by optimal orientation of the directional receiver antenna at the receiving point was not taken into account. The reason for this may be objective difficulties associated with the fundamental impossibility of taking into account the digitized shape pattern of the receiver antenna in the framework of the used analytical approach.

At the same time, an analysis of relation Eq. (4) allows to conclude that maximizing the size of the $\Omega_{\mathrm{s}}$ area in task Eq. (3) by increasing the power of the desired signal source $P_{1}$ is limited by the hardware capabilities of the transmitter, as well as the reception conditions. A promising direction here is improving the directive diagram characteristics of the transmitter and receiver, which, as practice shows, can significantly increase the size of the area of resilient radiotelephony. An option to overcome the corresponding difficulties may be a numerical approach.

It is an approach that was used in study [11] to determine the boundaries of region Eq. (2) under the action of several ground sources of additive frequencyconcentrated radio interference. It was assumed that the transmitter antenna had an omnidirectional directive diagram, and the radiation of radio interference sources was

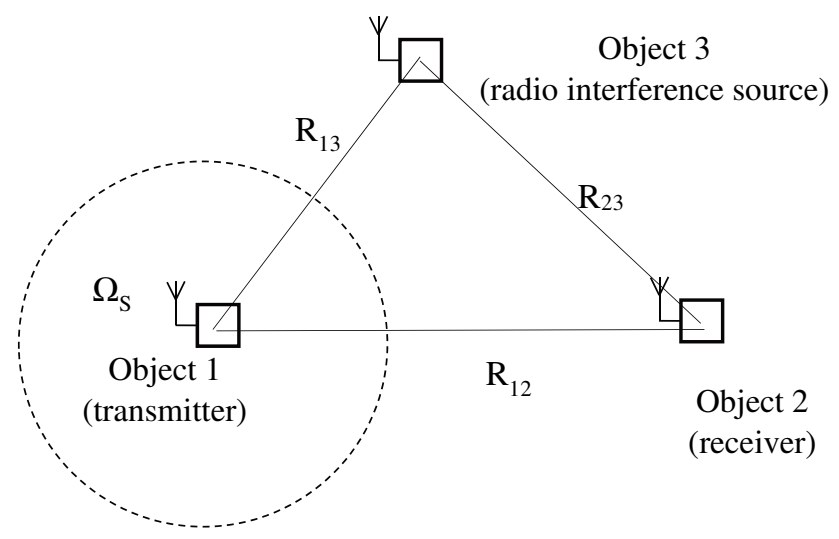

Fig. 1 Single source radio interference 
also non-directional. The suppression coefficient Eq. (1) of the transmitter desired signal at the input of the receiver with a two-dimensional directive diagram $G_{2}(\theta)$ of a beam-producing antenna is obtained in the following form:

$$
K_{\text {sup }}=\frac{R_{12}^{2}}{P_{1} G_{2}\left(\theta_{21}-\theta_{2}\right)} \sum_{i=1}^{K} \frac{P_{3_{i}} G_{2}\left(\theta_{23_{i}}-\theta_{2}\right)}{R_{23_{i}}^{2}}
$$

where:

$\theta_{21}$ - the azimuth of the receiver antenna to the signal transmitter,

$\theta_{2}-$ the signal receiver antenna azimuth,

$P_{3_{i}}$ - the radio interference $i^{\text {th }}$ source power,

$R_{23_{i}}$ - the distance from the receiver antenna to the $i^{\text {th }}$ interference source,

$\theta_{23_{i}}$ - the receiver antenna azimuth to $i^{\text {th }}$ interference.

It is shown that minimization of the suppression coefficient for a receiver located at a point $(x, y)$ can be achieved by choosing the optimal azimuth $\theta_{2}{ }^{*}$ of the receiver beam antenna system:

$$
K_{\text {sup }}\left(x, y, \theta_{2}^{*}\right) \rightarrow \min
$$

Using the numerical approach makes it possible to determine the values of the suppression coefficient Eq. (5) as a function of spatial coordinates $K_{\text {sup }}(x, y)$ with known placement parameters and properties of radiotelephony objects. Therefore, in the study [11], the problem of determining the boundaries of area Eq. (2) was formulated as a search for points of a level line $K_{\text {th }}$ in a scalar field (isolines)

$$
I_{K_{\text {th }}}=\left\{(x, y) \mid K_{\text {sup }}\left(x, y, \theta_{2}^{*}\right)=K_{\text {th }}\right\}
$$

The analysis of resources for computer visualization of level lines within the framework of task Eq. (7) makes it possible to draw the following conclusions [12]:

- the modern principle of interactivity, which imposes severe restrictions on the execution time of a program, makes the complete enumeration procedure unacceptable for task Eq. (7),

- well-known algorithms used in various application software packages (MatLab, Mathcad, Beta-Soft, etc.) cannot be used in other software systems, because their implementation procedures are not available, and it is impossible to scale the visualization area in the proportions suitable to real ones,

- the most popular algorithm for visualizing of two-dimensional scalar field is Marching Squares algorithm [13]. It is its versatility. The disadvantages of this method include the necessity to calculate the field values at all nodes of the initial regular grid, the presence of ambiguous cases when classifying fragments of the isoline and ensuring the isolation of the contour.

The disadvantage of the method proposed in the study [11] is the use of a twodimensional directive diagram of the receiver antenna in the radiotelephony model. It gives worse results obtained in the case of work with sources of interference located at an altitude. The solution of the problem Eq. (7) can be made more accurate by taking into account the three-dimensional properties of the directive diagram of the receiver antenna. Wherein the sources of interference will be located at different heights. To confirm the reliability of the calculations, it is advisable to experimentally study the 
shape and size of the noise-resistant radio exchange zone for the popular UHF/VHF radio communication equipment.

The purpose and objectives of the study are to develop a quick and suitable method for determining the boundaries of the noise-resistant radiotelephony area of the radio communication means of UHF/VHF band using beam antenna systems. To achieve this goal, it is necessary to solve the following tasks:

- improve the model of radio communication channel of UHF/VHF band by taking into account the elevation angle of interference sources located at different altitudes,

- to propose a numerical method for determining the boundaries of the noiseresistant radiotelephony area of maximum size, using an improved model of the radio communication channel, which is unambiguous and low laborious,

- to verify experimentally the reliability of the theoretical results obtained for widely used UHF/VHF band radio communication equipment operating in a real radio interference environment,

- to assess the effectiveness of the results.

\section{Research Objective of Determining the Boundaries of the Noise- resistant Reception Area}

\subsection{Three-dimensional Directive Diagrams of Antenna Arrangement}

To build an improved model of the UHF/VHF band radio communication channel, it is necessary to calculate the spatial function of the field strength of the antenna arrangement in polar coordinates $G(\theta, \varphi)$, where $\theta$ is the azimuth angle and $\varphi$ is the angular attitude. The pattern shape of the antenna system, if appropriate drawings are available, can be calculated using software tools for modeling three-dimensional electromagnetic fields, such as HFSS Ansoft [14]. Fig. 2 shows an example of a dipole antenna in HFSS Ansoft software calculation [12]. It is assumed that such a pattern shape in this task has a transmitter signal and the interference sources.

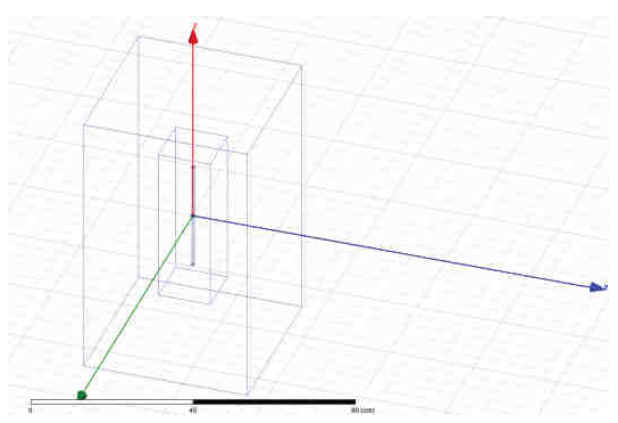

a)

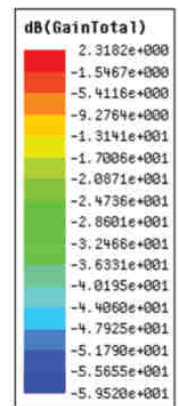

$5.9520++801$

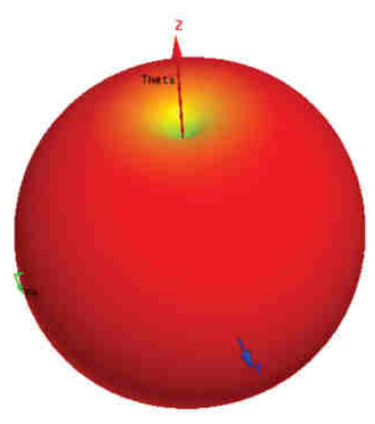

b)

Fig. 2 Calculation of the directive diagram for dipole antenna a) image of a dipole antenna model placed in a box $b$ ) pattern shape of nonresonant antenna arrangement

To protect the receiver from radio interference, any directional antenna or structure may be used, which includes a screen that defines the corresponding directive diagram. Fig. 3 shows an example of calculating the directive diagram of a beam 
antenna arrangement consisting of a dipole antenna of a radio facility Kenwood TK270 and a metal screen.

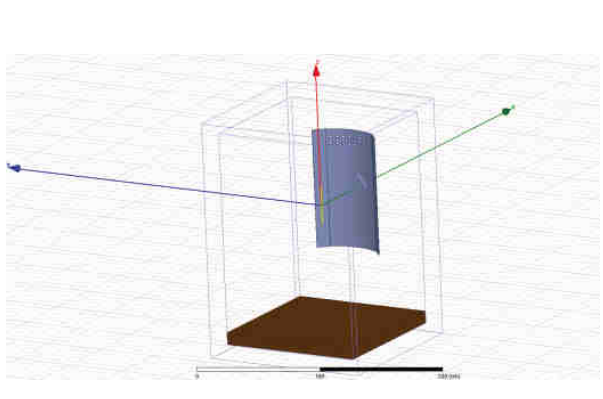

a)

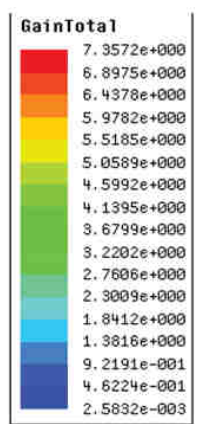

$5832 e-003$

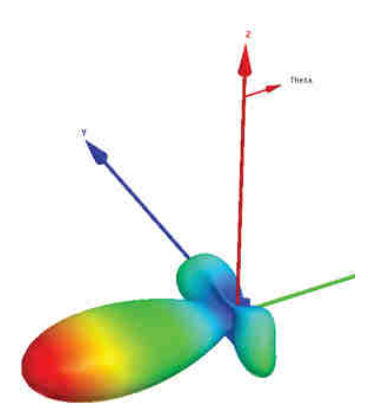

b)

Fig. 3 Calculation of the directive diagram for a dipole antenna equipped with a screen a) image of a dipole antenna model and a screen placed in a box $b$ ) directive diagram form of a beam antenna arrangement

Often normalized sections $D_{\mathrm{g}}(\theta), \theta \in\left[0^{\circ}, 360^{\circ}\right]$ and $D_{\mathrm{v}}(\varphi), \varphi \in\left[-90^{\circ}, 90^{\circ}\right]$ in the horizontal and vertical planes are used to describe directive diagrams. In this case, a three-dimensional normalized directive diagram of the antenna arrangement can be obtained as follows:

$$
G(\theta, \varphi)=D_{\mathrm{g}}(\theta) D_{v}(\varphi)
$$

Further, it will be assumed that in the considered problem, the signal receiver is equipped with a beam antenna arrangement with a digitized three-dimensional directive diagram.

\subsection{Model of UHF/VHF Band Radio Communication Channel under the Influence of an Interference System}

Consider the jamming circuit at the input of the radio receiver with a beam antenna system under conditions of several ground sources of additive frequency-concentrated radio interference (Fig. 4). The model UHF/VHF band radio communication channel contains objects with the following parameters.

Object 1 - the signal transmitter with power $P_{1}$ with nonresonant antenna at a point with coordinates $\left(x_{1}, y_{1}, z_{1}\right)$. The normalized directive diagram of the transmitter antenna is described by a function $G_{1}(\theta, \varphi)=1$.

Object 2 - the signal receiver with a beam antenna system at a point with coordinates $\left(x_{2}, y_{2}, z_{2}\right)$. The normalized radiation pattern of the transmitter antenna is described by the $G_{2}(\theta, \varphi)$ function obtained with calculation.

Object $3-S_{i}\left(x_{3_{i}}, y_{3_{i}}, z_{3_{i}}, P_{3_{i}}\right), i=1 \ldots K$. the radio interference source system with corresponding coordinates and radiation power. Radiation from radio interference sources is non-directional, therefore $G_{3_{i}}(\theta, \varphi)=1, i=1 \ldots K$.

According to Eq. (5), the suppression coefficient, taking into account the threedimensional directive diagram of the receiver antenna 


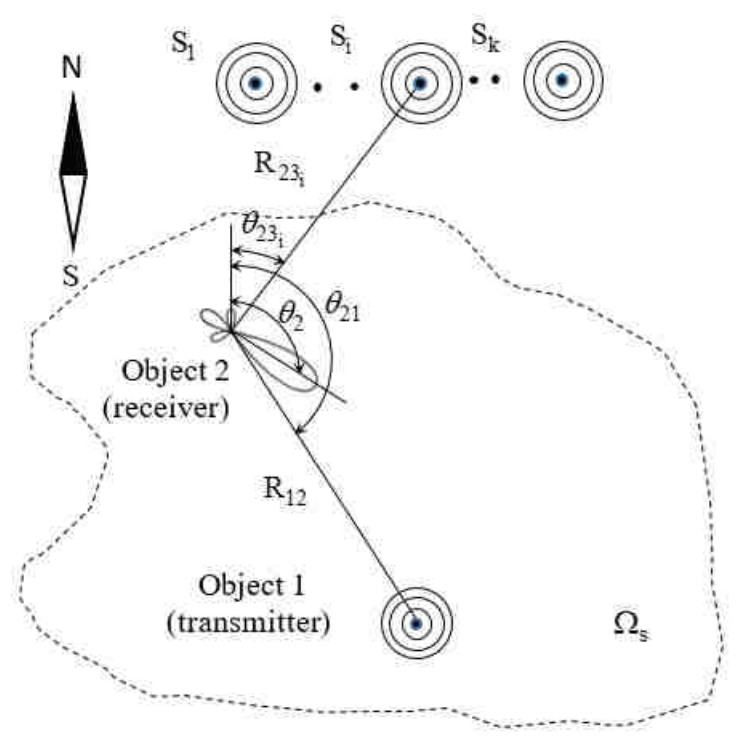

Fig. 4 Using a beam antenna system of receiver to protect against interference

$$
K_{\text {sup }}=\frac{R_{12}^{2}}{P_{1} G_{2}\left(\theta_{21}-\theta_{2}, \varphi_{21}-\varphi_{2}\right)} \sum_{i=1}^{K} \frac{P_{3_{i}} G_{2}\left(\theta_{23_{i}}-\theta_{2}, \varphi_{23_{i}}-\varphi_{2}\right)}{R_{23_{i}}^{2}}
$$

where $R_{12}=\sqrt{\left(x_{1}-x_{2}\right)^{2}+\left(y_{1}-y_{2}\right)^{2}+\left(z_{1}-z_{2}\right)^{2}}-$ is the distance from the transmitter to the receiver;

$$
\begin{aligned}
& \theta_{21}=\arctan \frac{\left|x_{2}-x_{1}\right|}{\left|y_{2}-y_{1}\right|}-\text { the azimuth from receiver to transmitter, } \\
& \varphi_{21}=\arctan \frac{\left|z_{1}\right|}{R_{12}}-\text { the angular altitude from receiver to transmitter, } \\
& \theta_{2}, \varphi_{2}-\text { the azimuth and angular altitude of the beam antenna of the receiver, } \\
& R_{23_{i}}=\sqrt{\left(x_{2}-x_{3_{i}}\right)^{2}+\left(y_{2}-y_{3_{i}}\right)^{2}+\left(z_{2}-z_{3_{i}}\right)^{2}}-\text { the distance from the }
\end{aligned}
$$
transmitter to the $i^{\text {th }}$ radio interference source,

$$
\theta_{23_{i}}=\arctan \frac{\left|x_{2}-x_{3_{i}}\right|}{\left|y_{2}-y_{3_{i}}\right|}-\text { the azimuth from the receiver to the } i^{\text {th }} \text { radio }
$$

interference source,

$$
\varphi_{23_{i}}=\arctan \frac{\left|z_{3_{i}}\right|}{R_{23_{i}}}-\text { the angular altitude from receiver to } i^{\text {th }} \text { radio interference }
$$
source. 


\subsection{Boundary Determination of the Error-free Reception Area}

Eq. (9) makes it possible to calculate the suppression coefficient at any point in the operational space. An example of calculating the scalar field $K_{\text {sup }}(x, y)$ for the case of a nonresonant receiver antenna $G_{2}(\theta, \varphi)=1$ is shown in Fig. 5.

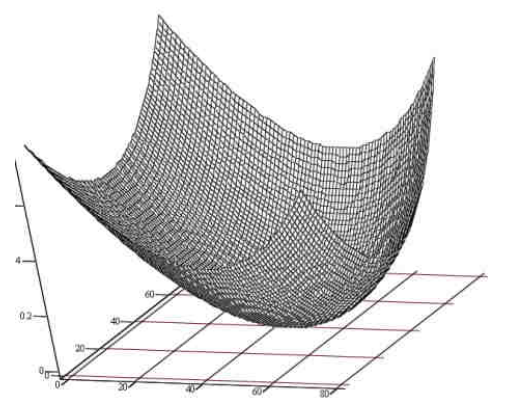

a)

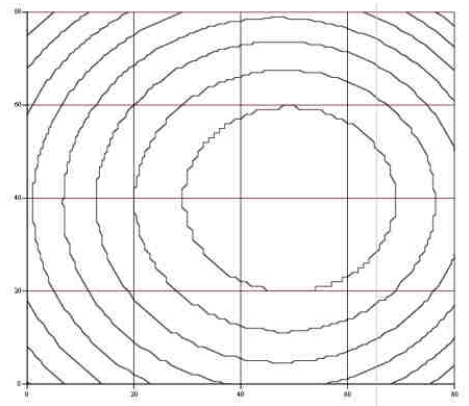

b)

Fig. 5 The spatial coordinate function $K_{\text {sup }}(x, y)$ for the case of a nonresonant receiver antenna

For the case of a beam antenna system of the receiver at a given transmitter power and interference sources, the condition for noise-resistant operation Eq. (1) at a point with $(x, y)$ coordinates depends mainly on the antenna orientation:

$$
K_{\text {sup }}\left(x, y, \theta_{2}, \varphi_{2}\right) \leq K_{\text {th }}
$$

In order to provide the most complete protection of the radiotelephony at each point of the noise-resistant radiotelephony area Eq. (2), it is advisable to calculate the optimal azimuth $\theta_{2}{ }^{*}$ and the optimal angular altitude $\varphi_{2}^{*}$ of the beam antenna system

$$
\theta_{2}^{*}, \varphi_{2}^{*}=\underset{\theta_{2} \in \Theta, \varphi_{2} \in \Phi}{\arg \min } K_{\text {sup }}\left(x, y, \theta_{2}, \varphi_{2}\right)
$$

where $\Theta \in\left[0^{\circ}, 360^{\circ}\right], \Phi \in\left[-90^{\circ}, 90^{\circ}\right]$.

The optimal orientation of the receiver antenna system in azimuth and angular altitude significantly changes the surface shape of the spatial coordinate function Eq. (10). Fig. 6 shows the results of calculating the scalar field $K_{\sup }\left(x, y, \theta_{2}{ }^{*}, \varphi_{2}{ }^{*}\right)$ for the case of a beam receiver antenna, taking into account Eq. (11).

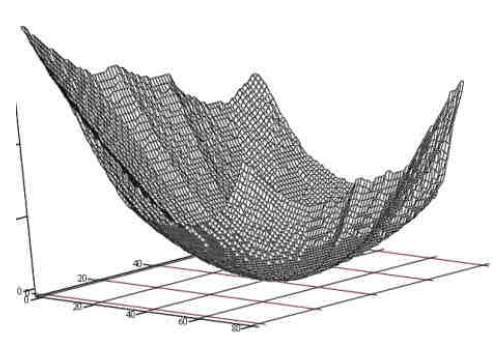

a)

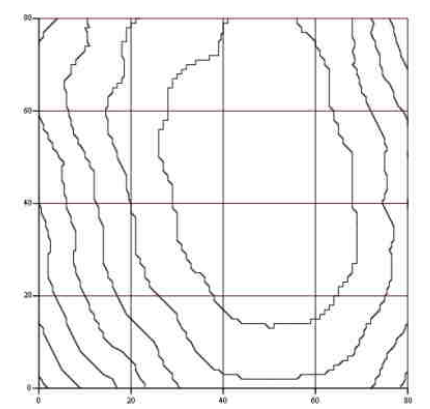

b)

Fig. 6 The spatial coordinate function $K_{\text {sup }}(x, y)$ for the optimal orientation of the beam receiver antenna 
Taking into account Eqs (10) and (11), the noise-resistant radiotelephony area of the maximum size for the UHF/VHF band radio communication channel with bean antennas system can be considered an area

$$
\Omega_{\mathrm{s}}=\left\{\forall(x, y) \in \Omega \mid K_{\text {sup }}\left(x, y, \theta_{2}^{*}, \varphi_{2}^{*}\right) \leq K_{\text {th }}\right\}
$$

Accordingly, the boundaries of the noise-resistant radiotelephony area Eq. (12) belong to the isolines of level $K_{\mathrm{th}}$, or to the geometrical place of the points with coordinates $(x, y)$ located at the altitude $K_{\text {th }}$ and have other points with different altitudes in any surroundings:

$$
I_{K_{\mathrm{th}}}=\left\{\begin{array}{l}
(x, y) \mid K_{\text {sup }}\left(x, y, \theta_{2}^{*}, \varphi_{2}^{*}\right)=K_{\mathrm{th}}, \\
\forall \varepsilon>0: \exists\left(x^{\prime}, y^{\prime}\right):\left|\left(x^{\prime}, y^{\prime}\right),(x, y)\right|<\varepsilon, K_{\text {sup }}\left(x^{\prime}, y^{\prime}, \theta_{2}^{*}, \varphi_{2}^{*}\right) \neq K_{\mathrm{th}}
\end{array}\right\}
$$

To calculate the point position of the isolines $I_{K_{\mathrm{th}}}$, it is necessary to develop a simple and effective numerical method that is unambiguous and low laborious.

\section{The Algorithm for Determining the Area of Noise-resistant Radiotelephony}

To calculate the coordinates of the isolines points Eq. (13), the fact that it is necessary to find only one level line, as well as the fact that in the plane $x 0 y$ the point of placement of the signal transmitter $\left(x_{1}, y_{1}\right)$ is always within the area $\Omega_{\mathrm{s}}$ is used.

The way out from this starting point to the boundary of the noise-resistant radiotelephony area is ensured by using the principle of the Lee wavelet algorithm [15]. The pixel matrix of the operational map image as an interference source will be considered.

Each pixel in the map image represents a cell of an interference source. To solve the problem of calculating the boundary of the noise-resistant area Eq. (13), the following modification of the wavelet algorithm is proposed.

Algorithm. Determine the boundaries of the stable radiotelephony area of mobile radio communication with optimal orientation three-dimensional radiation pattern of the receiver antenna (Fig. 7).

Input: $\left(x_{1}, y_{1}, z_{1}\right)$ : integer; $/ *$ Coordinate of signal transmitter */

$P_{1}$ : integer; /* Transmitted power of signal $* /$

$\left(x_{2}, y_{2}, z_{2}\right)$ : integer; /* Coordinate of signal */

$G_{2}(\theta, \varphi)$, /*directive diagram function of receiver's antenna */

$S_{i}\left(x_{3_{i}}, y_{3_{i}}, z_{3_{i}}, P_{3_{i}}\right), i=1 \ldots K:$ integer; /*Parameters of the sources of radio interference */

Output: Izoline: array[1...Li] of record $x, y:$ integer; /* Isoline point coordinates */

The complexity of the proposed algorithm is reduced in comparison with Marching Squares algorithm due to the fact that the calculation of the suppression coefficients $K_{\text {sup }}(x, y)$ is performed not at all points on the map, but only at the points of wave propagation. 


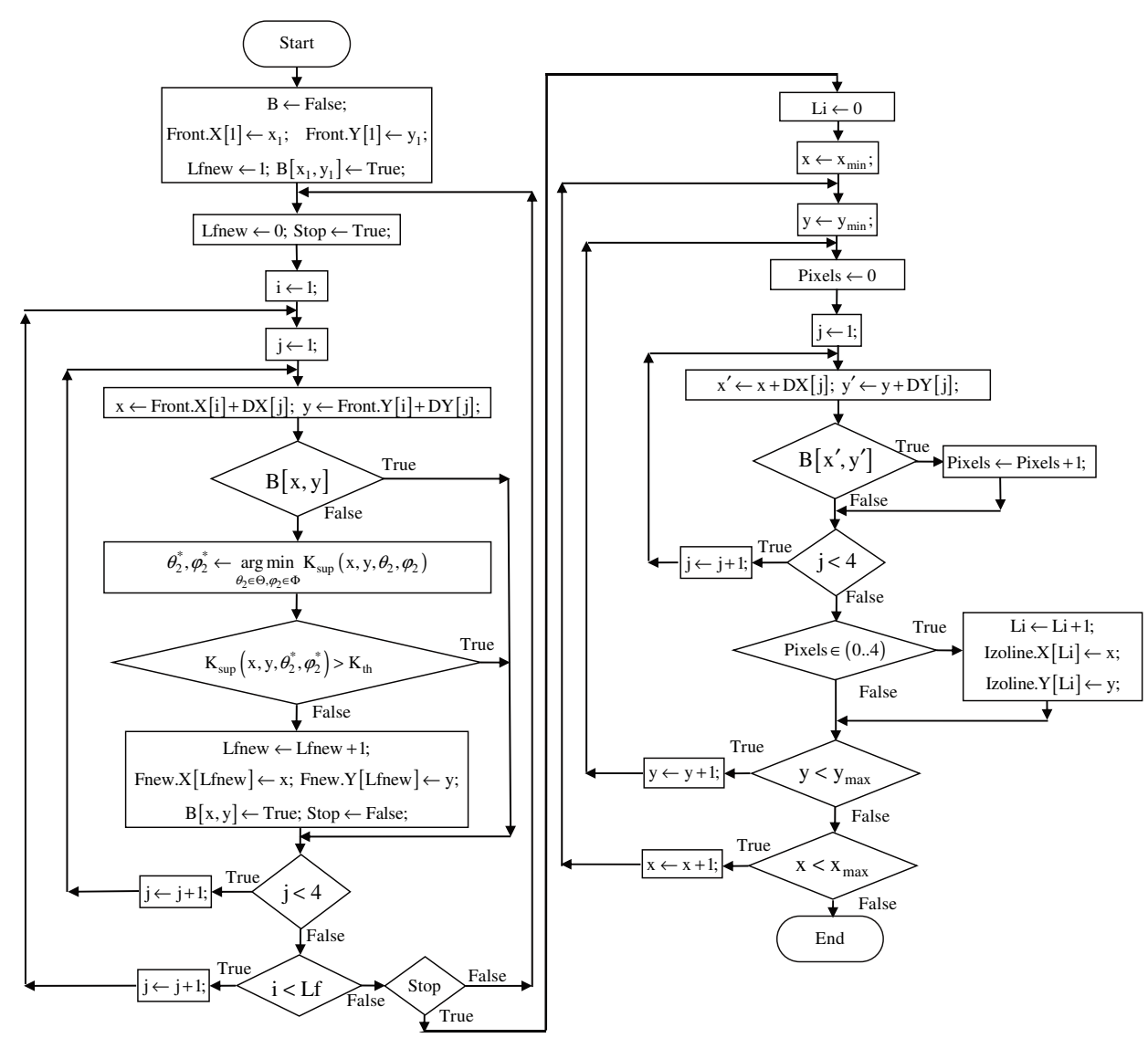

Fig. 7 Algorithm for determining the boundaries of the stable radiotelephony area

\section{Validation of Simulation Results}

The above-described algorithm for determining the boundaries of the stable radiotelephony area of mobile radio communication using directional antennas in the presence of real interference is implemented in a software tool to support the fast organization of secure radiotelephony. Omitting the interface part of the developed program, the results of calculations that demonstrate the adequacy of the proposed method are considered.

Fig. 8 presents the results of calculating the boundaries of the noise-resistant radiotelephony area with the nonresonant antenna system of the receiver for two cases of different combinations of signal source power and interference.

Case 1 . The energy potential of the interference source exceeds the power of the signal transmitter. The jamming reception area is inside the circle on the left.

Case 2. The energy potential of the interference source is small. The noiseresistant area is the entire space of the card except the suppression area bounded by a circle to the right. 


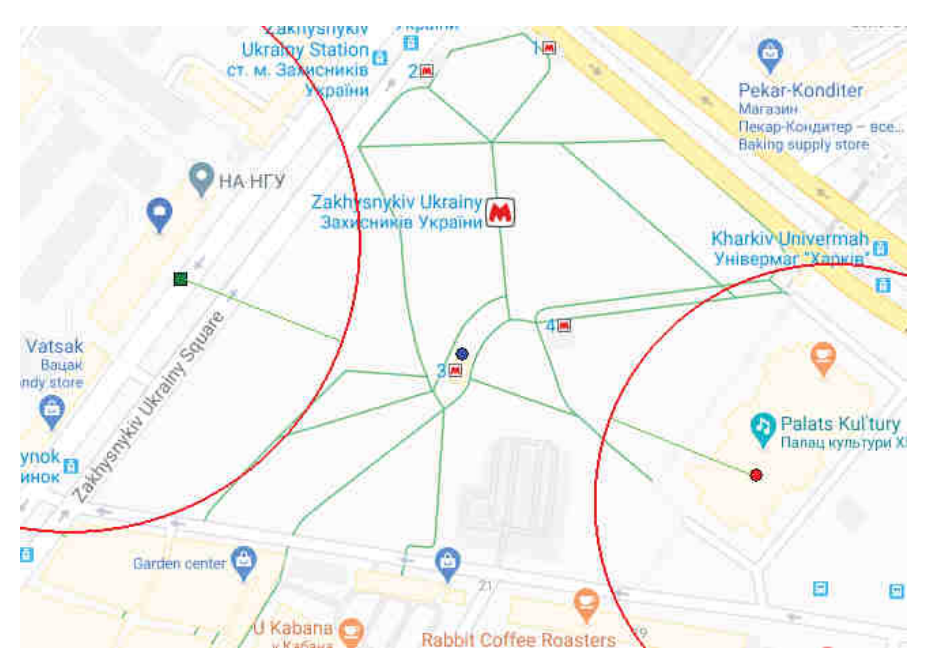

Fig. 8 Calculation of the boundaries of noise-resistant radiotelephony areas for a receiver with a buggy-whip antenna

Comparing the shape of the noise-resistant radiotelephony areas in Fig. 8 with the corresponding results of [1], it is possible to conclude that the results of calculations are consistent with the data obtained for these particular cases by the analytical method. Further, to assess the reliability of the results obtained, a comparative analysis of simulation data and experimental data for Mototrbo ${ }^{\mathrm{TM}}$ DP4800 radio equipment [16] was performed. The Kenwood TK-8302 radio facility [17] was used as a source of interference.

The experiment was conducted in two stages:

- determination of the noise-resistant radiotelephony area using nonresonant antenna arrangement,

- determination of the noise-resistant radiotelephony area using beam antenna system.

At the stage 1, the research facility consisted of three objects (Fig. 9).

Object 1. A digitally mode stationary immovable signal transmitter equipped with a rotary measuring mechanism and a laser distance meter (Mototrbo ${ }^{\mathrm{TM}}$ DP4800 radio facility).

Object 2. Mobile signal sink, which operates in digital mode (Mototrbo $^{\mathrm{TM}}$ DP4800 radio facility).

Object 3. Immovable interference source operating in analogue mode (Kenwood TK-8302 radio facility).

Prior to the experiment, the following turnings were set up:

- configuration of object 1 to the operation frequency $412.025 \mathrm{MHz}$ in digital mode with an output rating $1 \mathrm{~W}$,

- configuration of object 3 to the operation frequency $412.025 \mathrm{MHz}$ in analogue mode with an output rating $15 \mathrm{~W}$.

The experiment was performed for 10 samples of the Mototrbo ${ }^{\mathrm{TM}} \mathrm{DP} 4800$ radio facility, used as a mobile object 2. During the experiment, the mobile object 2 was removed from the object 1 in the direction determined by the azimuth of the rotation measurement mechanism. For certain values of the signal strength $P_{1}$ and the 


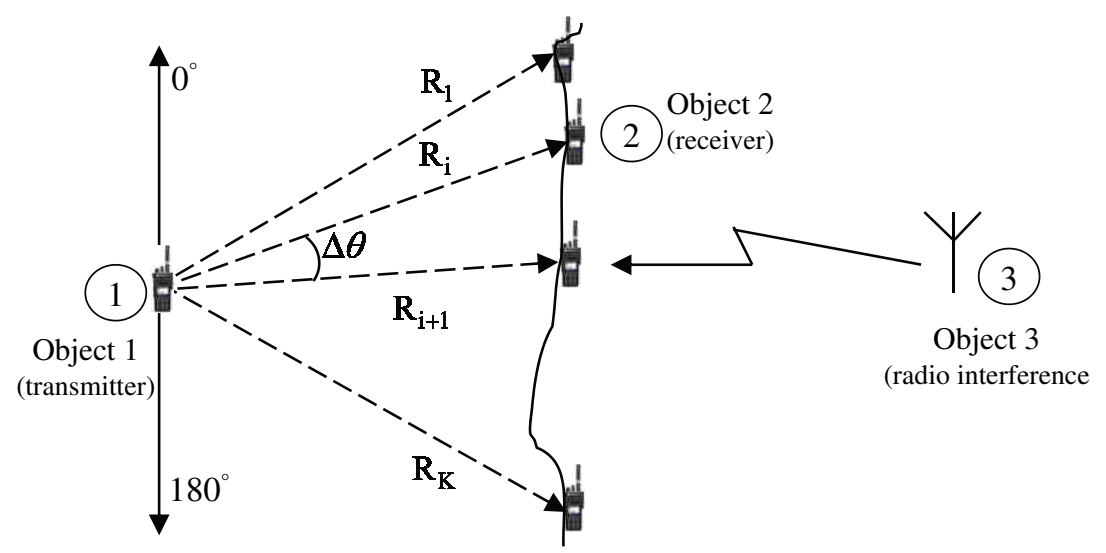

Fig 9 Experiment scheme on stage 1

countermeasure $P_{3}$, the distance $\tilde{R}_{\mathrm{e}_{i}}, i=1 \ldots K$ from object 1 to object 2 was determined using a laser meter, at which the coefficient $K_{\text {sup }}$ reached a threshold rate sufficient for the object 2 to switch suppression mode. The obtained distance determined the boundary point of the noise-resistant radiotelephony area at a certain azimuth relative to object 1 . The pitch of the azimuth angle is $10^{\circ}\left(\Delta \theta=10^{\circ}\right)$. Further, the average distance $R_{\mathrm{e}_{i}}, i=1 \ldots K$ values were obtained for the group of samples of Mototrbo $^{\mathrm{TM}}$ DP4800 radio equipment function as object 2.

In Fig. 10, for the signal strength $P_{1}=1 \mathrm{~W}$ and countermeasure $P_{3}=15 \mathrm{~W}$ values, the averaged experimental $R_{\mathrm{e}_{i}}, i=1 \ldots K$ values are compared with the distances $R_{\mathrm{t}_{1}}, i=1 \ldots K$ obtained as a result of computer simulation in stage 1 .

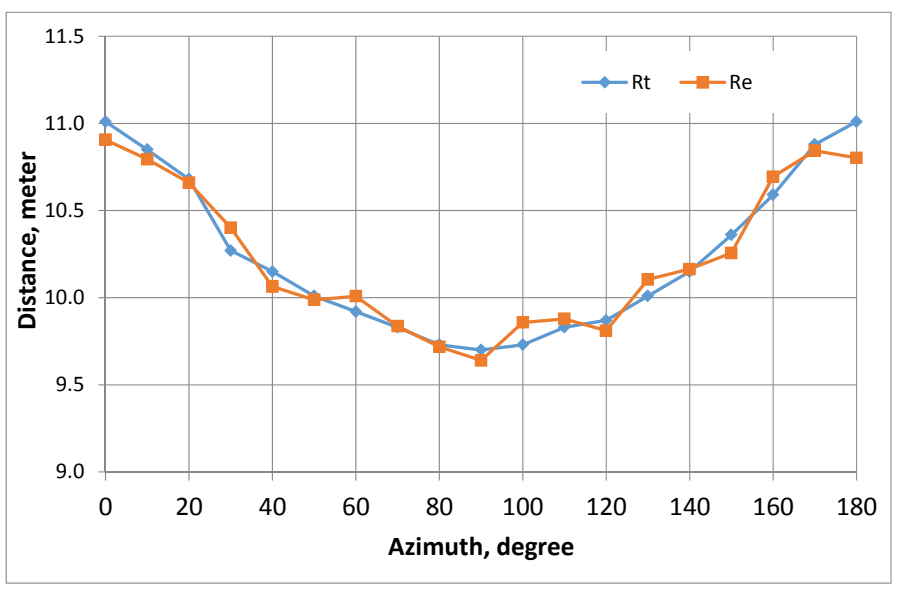

Fig. 10 Comparison of stage 1 experimental data $R_{\mathrm{e}}$ with computer simulation data $R_{\mathrm{t}}$

The experimental scheme on the stage 2 is shown in Fig. 11. The movable signal sink (object 2) was equipped with a beam antenna system in the form of a screen. 


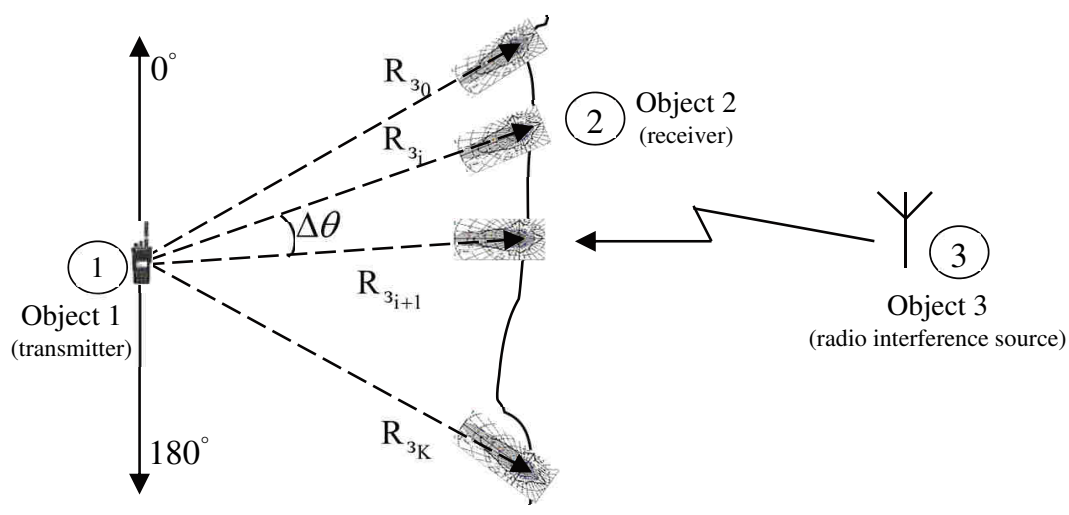

Fig. 11 Experiment scheme on stage 2

Before the experiment, pre-settings similar to those described above were made. The experiment was performed for 10 Mototrbo $^{\mathrm{TM}}$ DP4800 samples used as a mobile object 2. During the experiment, the movable object 2 was removed from the object 1 in the direction determined by the azimuth of the rotary measurement mechanism. At each point of the mechanical trajectory, the antenna arrangement of object 2 was oriented to object 1 . For certain values of signal strength $P_{1}$ and countermeasure $P_{3}$, a distance meter $\tilde{R}_{\mathrm{e}_{i}}, i=1 \ldots K$ was determined from the object 1 to object 2 using a laser meter, at which the coefficient $K_{\text {sup }}$ reached a threshold value sufficient for the object 2 to switch the suppression mode. The obtained distance determined the boundary point of the noise-resistant radiotelephony area at a certain azimuth relative to object 1 . The pitch of the azimuth angle is $10^{\circ}\left(\Delta \theta=10^{\circ}\right)$. Further, the average distance $R_{\mathrm{e}_{i}}, i=1 \ldots K$ values were obtained for the group of samples of Mototrbo ${ }^{\mathrm{TM}}$ DP4800 radio equipment function as object 2. In Fig. 12, for the signal strength $P_{1}=1$ $\mathrm{W}$ and countermeasure $P_{3}=15 \mathrm{~W}$ values, the averaged experimental values of $R_{\mathrm{e}_{i}}, i=1 \ldots K$ are compared with the distances $R_{\mathrm{t}_{1}}, i=1 \ldots K$ obtained as a result of computer simulation on the stage 2 .

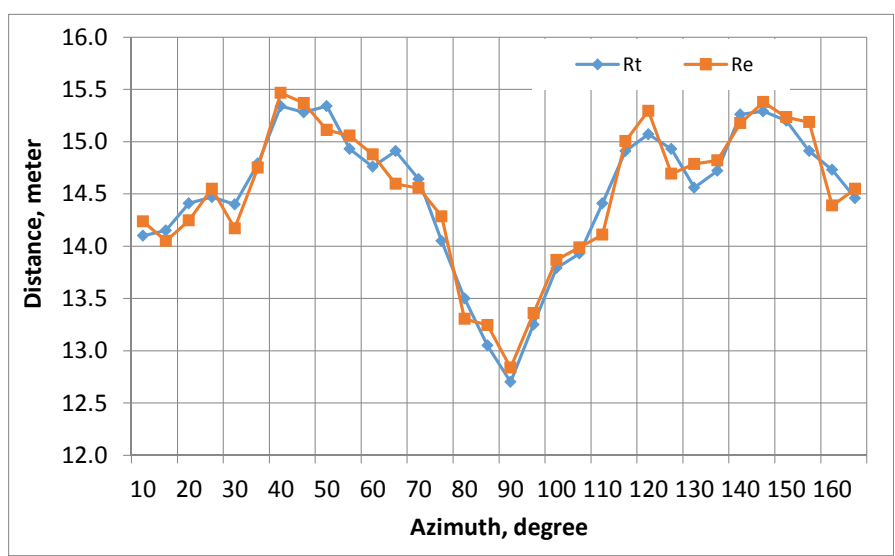

Fig. 12 Comparison of stage 2 experimental data $R_{\mathrm{e}}$ with computer simulation data $R_{\mathrm{t}}$ 
The results of computer simulations at all stages demonstrate close agreement with experimental data. The variation range of the absolute errors of measurement is $\Delta=(0.15-0.56) \mathrm{m}$ for a confidence coefficient of 0.96 . The relative error is $\delta=(1.49$ $5.63) \%$, and it allows stating that the results of mathematical modeling are adequate.

Therefore, it can be concluded that the obtained simulation analysis results are validated during the assessment of the radiotelephony noninterference by radio communication using additional beam antenna systems.

\section{Assessment of Proposed Approach Effectiveness}

In the developed support program for the fast organization of secure radiotelephony, calculations are carried out in conjunction with a map of the area. Consider an example of calculations confirming the effectiveness of the proposed approach. The influence of the beam antenna system orientation method on the form and size of the noise-resistant radiotelephony area can be estimated from the calculation results for a particular case of the operational situation in urban conditions (Fig. 13). A single source of interference is located at the height of the third floor of a detached house.

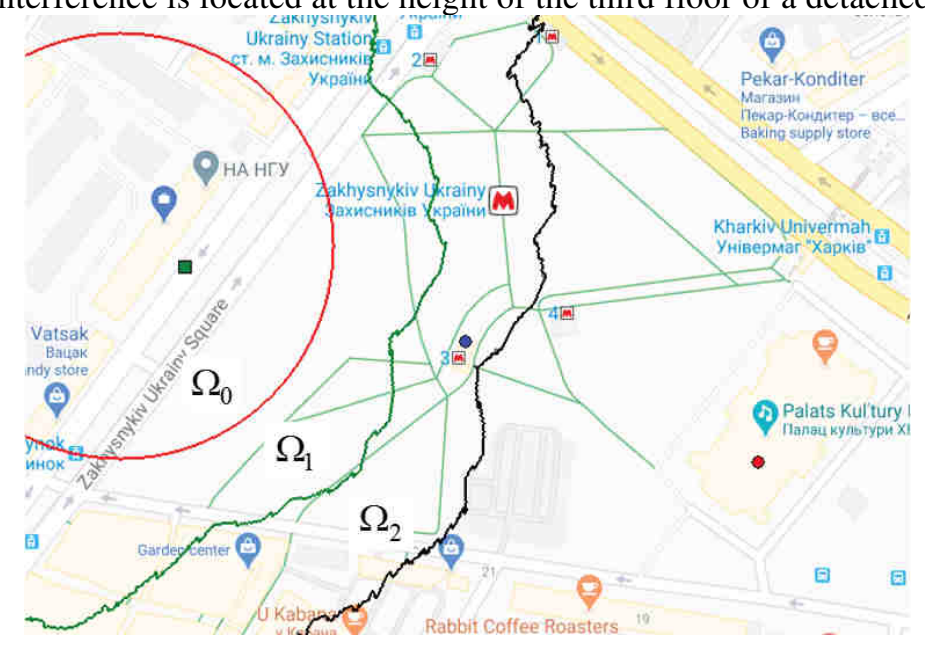

Fig. 13 Noise-resistant radiotelephony areas for various methods of orienting the beam antenna system of the receiver

Noise-resistant radiotelephony area $\Omega_{0}$ for the case of a receiver with a buggywhip antenna is located inside the area bounded by a circle. The boundaries of the area $\Omega_{1}$ were obtained, when the receiver uses a beam antenna system with an optimal orientation in the azimuth angle. The boundaries of the noise-resistant radiotelephony area were obtained using Eq. (10) when the receiver uses a beam antenna system with optimal orientation in azimuth and angular altitude.

The corresponding values of the coverage coefficient of the area Eq. (3) on the map are $K_{\operatorname{cov} 0}=0.18, K_{\operatorname{cov} 1}=0.34, K_{\text {cov } 2}=0.5$. The increase in the coverage factor of the area on the map for the maximum noise-resistant radiotelephony area $\Omega_{2}$ is 2.8 times towards $\Omega_{0}$ and 1.5 times towards $\Omega_{1}$ due to the optimal orientation of the beam antenna of the receiver system at each point of the map not only in the azimuth angle, but also in angular altitude. 


\section{Conclusions}

A method for determining the boundaries of the maximum area of stable radio reception in the UHF/VHF band for mobile radio communication equipment is proposed.

The method uses an improved model of a radio communication channel under the influence of a system of interference sources located at different altitudes. The model requires a three-dimensional diagram of the signal receiver antenna which is necessary for the usage of the model.

The problem of determining the boundaries of the maximum size area of stable radio reception in the form of finding a single isoline in a scalar field is formulated. To search for such an isoline, a modification of the wave algorithm using an improved model of the radio channel has been proposed. Increasing the size area of stable radio reception is achieved by optimal orientation of the signal receiver antenna at each point.

Experimental confirmation of the results was obtained for the widely used radio communication UHF/VHF band (Mototrbo ${ }^{\mathrm{TM}}$ DP4800 and Kenwood TK-8302 radio equipments) operating in real radio interference. The results of computer simulations demonstrate close agreement with experimental data.

The variation range of the absolute errors of measurement is $\Delta=(0.15-0.56) \mathrm{m}$ for a confidence coefficient of 0.96 . The relative error is $\delta=(1.49-5.63) \%$, and it allows stating that the results of mathematical modeling are adequate.

A computer program has been developed to support the fast organization of secure radiotelephony of the radio communication UHF/VHF band.

The effectiveness of the proposed method is confirmed by an increase in the area of the noise-resistant radiotelephony area by 2.8 times towards the use of a mobile radio with a conventional buggy-whip antenna. For the case of optimal orientation of the beam antenna system of the receiver at each point of the map, only in the azimuth angle additional optimization in angular altitude gives a gain of 1.5 times.

The method allows receiving a real noise-resistant radiotelephony area, whose form depends on the characteristics and orientation of the antennas of the radio communication means and the quantity, power and spatial location of the interference source.

\section{Acknowledgement}

The work presented in this paper has been supported by the Main Directorate of the National Guard of Ukraine (research project No. DR 0118U004670).

\section{References}

[1] KUPRIYANOV, A.I. Electronic Warfare (in Russian). Moscow: University book, 2013. 360 p. ISBN 978-5-9502-0501-9.

[2] KUPRIYANOV, A., OVCHINSKY, A., BETSKOV, A. and KOROBKO, V. Radio-Electronic Warfare as a Conflict Interaction in the Information Space. In: KRAVETS, A., ed. Big Data-driven World: Legislation Issues and Control Technologies. Studies in Systems, Decision and Control. Berlin: Springer, 2019, p. 173-179. ISBN 978-3-030-01357-8. 
[3] YOHOV, O. Method of Optimizing the Protection from the Radio Reconnaissance of the Radio Exchange in the Communication Networks of the Troops (Forces). Magyar Tudományos Journal, 2018, no. 14, p. 44-47. ISSN 1748-7110.

[4] Jamming Solutions [on line]. Samokov, Bulgaria: Samel-90 PLC [viewed 202004-07]. Available from: https://cutt.ly/TtHmsq2

[5] EMC within Power Plants and Substations [on line]. [Technical Brochure]. Working Group C4.208, 2013. 309 p. [viewed 2019-10-01]. Available from: https://cutt.ly/ltJy5Bz

[6] RACHIDI, F. and TKACHENKO, S. Electromagnetic Field Interaction with Transmission Lines: From Classical Theory to HF Radiation Effects. Southampton: WITpress, 2008. 288 p. ISBN 978-1-84564-063-7.

[7] DRAGAN, P. Advanced Modeling in Computational Electromagnetic Compatibility. New York: Wiley, 2007. 520 p. ISBN 978-0-47-003665-5.

[8] HTZ Warfare [on line]. Paris: ATDI. [viewed 2019-06-19]. Available from: https://cutt.ly/ZtJupgp

[9] EMC Analysis [on line]. Belvoir, Defense Acquisition University [viewed 202004-07]. Available from: https://cutt.ly/XtHRoC6

[10] KHARKEVICH, A.A. Interference Control (in Russian). Moscow: Fizmatgiz, 1963. 276 p. ISBN 978-5-9710-5339-2.

[11] MALIUK, V., YOHOV, O. and KUZMINICH, I. Method for Determining the Boundaries of a Stationary Radio Exchange Zone for Internal Troops in Radio Suppression (in Ukraine). Weapons Systems and Military Equipment, 2014, vol. 1, no. 37, p. 56-62. ISSN 1997-9568.

[12] MALIUK, V., YOHOV, O., KUZMINICH, I and SEVERINOV, A. Evaluation of Antijammingness of Channel of Radio Contact of Tactical Link of Management of Subdivisions of Internal Troops by a Simulation Technique (in Ukraine). Control, Navigation and Communication Systems, 2013, vol. 2, no. 26, p. 179-185. ISSN 2073-7394.

[13] Marching Squares [on line]. [viewed 2020-04-07]. Available from: https://cutt.ly/etHYEaq

[14] ANSYS HFSS. 3D Electromagnetic Field Simulator for RF and Wireless Design. [on line]. [viewed 2019-08-02]. Available from: https://cutt.ly/HtJrTD7

[15] Lee, C.Y. An Algorithm for Path Connections and Its Applications. IRE Transactions on Electronic Computers, 1961, vol. EC-10, no. 3, p. 346-365. DOI 10.1109/TEC.1961.5219222.

[16] Mototrbo ${ }^{\mathrm{TM}}$ DP4800/DP4801 Digital Portable Two-Way Radio [on line]. [viewed 2019-05-27]. Available from: https://cutt.ly/4tJyJrp

[17] Kenwood TK-8302(H) [on line]. [viewed 2019-05-27]. Available from: https://cutt.ly/btJyNpO 